\title{
La chapelle Montfalcon à la cathédrale de Lausanne
}

\section{Kérim Berclaz}

\section{OpenEdition}

Journals

Édition électronique

URL : http://journals.openedition.org/edl/1428

DOI : $10.4000 /$ edl. 1428

ISSN : 2296-5084

\section{Éditeur}

Université de Lausanne

\section{Édition imprimée}

Date de publication : 1 décembre 2018

Pagination : 241-258

ISBN : 978-2-940331-69-7

ISSN : 0014-2026

\section{Référence électronique}

Kérim Berclaz, "La chapelle Montfalcon à la cathédrale de Lausanne », Études de lettres [En ligne], 3-4 I 2018, mis en ligne le 15 décembre 2020, consulté le 17 décembre 2020. URL : http:// journals.openedition.org/edl/1428; DOI : https://doi.org/10.4000/edl.1428 


\section{LA CHAPELLE MONTFALCON À LA CATHÉDRALE DE LAUSANNE}

En 1504, Aymon de Montfalcon fonde une fastueuse chapelle sous la tour nord du massif occidental de la cathédrale. Les sources conservées montrent les moyens déployés par l'évêque dans sa fondation privée pour assurer sa mémoire (sépulture, legs et messes commémoratives), ainsi que la position stratégique qu'occupe cette chapelle au sein de l'espace ecclésial suite à d'importants travaux.

Un corpus exhaustif de testaments a récemment permis de découvrir les stratégies mémorielles des évêques de Lausanne entre 1301 et $1461{ }^{1}$. Cette chronologie, limitée par les aléas documentaires, excluait Aymon de Montfalcon, mort le 10 août 1517 et dont le testament - qui a probablement existé ${ }^{2}-$ a disparu. D'autres sources existent en revanche, qui contournent cette perte. Elles dévoilent l'intérêt que représente la célèbre fondation, en 1504, de la chapelle voulue par cet évêque et dédiée à saint Maurice et aux martyrs thébains ${ }^{3}$. Aymon de Montfalcon, prélat dont

I. K. Berclaz, Les voies de l'éternité.

2. Ibid., p. 28. Il n'est pas impossible que celui-ci ait disparu au moment où Sébastien de Montfalcon, neveu de l'évêque, fuit Lausanne suite à la Réforme instaurée à Lausanne en 1536, emportant avec lui une partie des archives épiscopales. Cf. J.-D. Morerod, Genèse d'une principauté épiscopale, p. 19 et G. Coutaz, "L’inventaire de 1394 des archives de l'évêque de Lausanne», en part. p. 115, n. 108.

3. Lors de la séance du 25 septembre 1504, les chanoines du chapitre accordent à Aymon de Montfalcon le droit de fonder et d'édifier une chapelle dans la cathédrale, et lui octroient un emplacement sous la tour nord du clocher en construction (qui ne connaîtra jamais d'achèvement): Die vero mercurii vigesimaquinta dicti mensis septembris [...] fuit R. D. episcopo per quos supra dominos capitulantes concess(us) locus infra dictam ecclesiam, videlicet quod possit [...] edificare et fundare capellam suam, et hoc in fine dicte 
l'importance à l'échelle du diocèse et de la cathédrale est bien connue ${ }^{4}$, agit comme ses prédécesseurs en matière de commémorations. Ainsi, il s'agira dans un premier temps de souligner comment une fondation privée, et de surcroît funéraire, devient un pôle d'attraction extrêmement important pour concentrer la mémoire du défunt grâce à différentes pratiques visant à sanctifier l'espace qui lui est consacré, participant par là même à la privatisation du lieu. Dans un deuxième temps, nous chercherons à éclairer la logique de l'emplacement de la chapelle dans l'édifice en fonction des pratiques liées à la piété et à la dévotion propre à la cathédrale au début du XVI siècle. Mémoire individuelle et insertion dans l'espace ecclésial constitueront ainsi les deux axes d'analyse ayant trait à la chapelle Montfalcon.

\section{Les sources à disposition}

Plusieurs documents permettent de pallier la disparition du testament d'Aymon de Montfalcon et de questionner sa fondation funéraire. Nous en avons retenu quatre:

- l'acte de fondation de la chapelle, rédigé entre le 2 et le 10 août 1517, qui donne dans les détails les modalités des commémorations quotidiennes souhaitées par Aymon (nombre de chapelains et leurs salaires annuels $)^{5}$;

- le compte-rendu de la visite des autels et des chapelles de la cathédrale en 1529 - celle de saint Maurice et des martyrs thébains étant inspectée le 8 décembre ${ }^{6}$. Ce document, bien connu des historiens et beaucoup commenté depuis la fin du XIXe siècle, renseigne sur le mobilier liturgique de la fondation et sur les services quotidiens voulus par l'évêque;

ecclesie sub novo simballatorio quod nond(um) est finitum. Cf. Archives cantonales vaudoises (ACV), Ac 13, fol. 3r-v, manual du chapitre de Lausanne, mercredi 25 septembre 1504.

4. M. Reymond, "Aymon de Montfalcon, évêque de Lausanne, 1491-1517». Cf. également l'article de B. Andenmatten dans ce même volume.

5. ACV, C Vb 993.

6. ACV, Ac 15/1, fol. 102r-105r et Ac 15/2, fol. 228v-234r (copie des 168 premiers folios du minutaire coté Ac 15/1; nous nous basons ici sur cette deuxième version). 
- le manual du chapitre de Lausanne (1504-1519) consignant les décisions importantes prises par les chanoines lors de séances hebdomadaires ${ }^{7}$. Cette source riche en informations sur la vie capitulaire indique les mesures relatives à la chapelle, à la mort et à la sépulture de l'évêque, ainsi qu'aux messes annuelles et leurs prix. Elle renseigne plus largement sur les multiples transformations que subit le massif occidental dès 1499 , et dont l'évêque, de concert avec le chapitre, sera largement responsable. C'est dans ce contexte de réaménagements que la chapelle s'insère;

- le nécrologe de la cathédrale, rédigé ou complété au début du $\mathrm{XVI}^{\mathrm{e}}$ siècle ${ }^{8}$. Ce livre commémoratif de la communauté des fidèles de l'Église lausannoise recense de nombreuses messes d'anniversaire et commémoratives à l'intention des bienfaiteurs de la cathédrale, parmi lesquels figure Aymon de Montfalcon.

\section{La chapelle d'Aymon de Montfalcon (1504): une vitrine pour l'éternité}

La fondation dédiée à saint Maurice et à la légion thébaine est un écrin privatisé pour manifester durablement l'existence d'Aymon. C'est là que l'évêque porte l'essentiel de son attention. La recherche d'une privatisation est d'ailleurs particulièrement claire dans son repli sur elle-même: du côté du vestibule, une paroi percée d'une porte en ferme l'accès, tandis qu'une grille en fer forgé clôt son entrée du côté de la nef ${ }^{9}$. Les sources illustrent donc avant tout à quel point cette chapelle représente un lieu funéraire et de commémoration. Le souvenir se manifeste ainsi sur deux plans: d'une part, ce qui a trait au visuel et au matériel avec la présence du tombeau et des legs spécifiques de l'évêque dans la chapelle; d'autre part, ce qui découle de l'immatériel avec les nombreuses messes commémoratives récitées durant l’année.

7. ACV, Ac 13.

8. J. Gremaud, «Nécrologe de l'église cathédrale de Lausanne», en part. p. 99, 104, 171,207 et 227.

9. ACV, Ac 15/2, fol. 230r-v: [...] una parva janua de dicto ligno nucis sumptuose composita, minusiata cum uno parvo guicheto seu parva fenestra, respondente a dicto latere novi magni portalis, ferrata a directo dicte parve janue in cadro dicti altaris reposita, etc. [...]. Aliunde dicta capella a parte navis ecclesie, in suo introitu, reperitur firmiter clausa ferratura seu talliis ferri a pede usque sursum, janua inibi existente et sera optimis. 
2.1. Le tombeau, le mobilier liturgique et le décor de la chapelle

L'ensevelissement d'Aymon de Montfalcon dans sa chapelle est certifié dans le manual du chapitre. Le mardi 11 août 1517, une notice nous apprend que l'on discute au sujet des funérailles dont l'évêque doit bénéficier; ce même jour, le corps est enseveli à l'intérieur de la chapelle:

Et exposuit qualiter herina die deffuncto quondam bone memorie reverendissimo domino Aymone de Montefalcone episcopo Lausannensi et principi, sicut eis placuit consilium petiit de modo eius corpus sepelliendi funeralibusque et obsequiis eius faciendi, qui fuit consultum, quod sepelliatur et obsequie ac funeralia fiant tam honorifice et ut melius fieri poterit qui egit gracias. [...] Consequenter, dicta die, finitis vesperis et vigiliis, ut decuit, fuit honorifice corpus prefati quondam reverendissimi d. Aymonis episcopi et principis infra dictam Lausannensem ecclesiam, videlicet in capella noviter per eum fondata inhumatum ${ }^{10}$.

La présence matérielle du tombeau est confirmée à deux reprises dans le nécrologe, le 14 janvier et le jour de la Sainte-Trinité, qui mentionne des processions générales observées par les chanoines «sur le tombeau du révérend seigneur de bonne mémoire et père dans le Christ Aymon de Montfalcon " ${ }^{11}$. Enfin, une chronique tardive de Jean-Baptiste Plantin atteste encore en 1656 la présence de son «effigie en bosse [qui se voyait encore] sur son tombeau " ${ }^{12}$. La représentation funéraire, vraisemblablement en ronde-bosse et associée au tombeau, se trouvait encore dans la chapelle plus d'un siècle après l'instauration de la Réforme en 1536 à Lausanne. Il serait faux de considérer cet événement comme facteur de destructions massives et incontrôlées de la part des protestants ${ }^{13}$. De même, il serait étonnant que les Bernois autorisent la destruction des monuments funéraires des évêques, représentants de l'autorité lausannoise dont ils sont les successeurs immédiats ${ }^{14}$. La conservation

Io. ACV, Ac 13, fol. 237r.

II. J. Gremaud, "Nécrologe de l'église cathédrale de Lausanne», p. 99 et 227.

I2. BCU, Ms., F. 1071 : J.-B. Plantin, De l'antiquité de la ville de Lausanne, p. 162.

13. Il a été démontré que les Bernois ont pris soin de consigner et de conserver systématiquement, au niveau documentaire, les pièces pouvant établir les possessions de l’Église de Lausanne et appartenant désormais aux Bernois. Cf. J.-D. Morerod, Genèse d'une principauté épiscopale, p. 19 sq.

I4. Nous remercions Gaëtan Cassina pour cette indication pertinente. 
de six effigies funéraires épiscopales dans la cathédrale en témoigne ${ }^{15}$. Aujourd'hui, celle d'Aymon de Montfalcon a disparu de la chapelle, mais il n'est pas impossible que l'une des effigies épiscopales préservées coïncide avec ce que nous savons du prélat. En effet, le gisant conservé aujourd'hui à l'angle sud-ouest du bras sud du transept correspond à plus d'un titre au tombeau du prélat: c'est un évêque, reconnaissable à sa mitre et aux restes de la hampe d'une crosse creusée contre sa poitrine. Pour des raisons stylistiques, chronologiques et typologiques, le rapprochement entre ce monument et celui d'Aymon est permis. L'analyse de Gaëtan Cassina renvoie à une représentation datable stylistiquement de la deuxième moitié du $\mathrm{XV}^{\mathrm{e}}$ siècle, voire du début de la Renaissance ${ }^{16}$; il s'agit de plus du seul prototype en ronde-bosse conservé - soit une technique décrite par Jean-Baptiste Plantin -, alors que les autres vestiges préservés dans la cathédrale et les sources d'archives concernant les monuments funéraires épiscopaux renvoient plutôt à des plates-tombes ou à des gisants en bas-relief; enfin, le matériau calcaire rappelle la pierre du Jura utilisée par les sculpteurs des statues de la galerie du portail Montfalcon ${ }^{17}$. Il n'est donc pas impossible que l'évêque ait pensé à faire construire son tombeau bien avant sa mort et les travaux du portail, afin de s'assurer de son exécution de son vivant.

La présence de cet important monument à l'intérieur de la chapelle contribuait fortement à l'évocation du corps et donc à réactiver le souvenir du défunt. La tombe rendait visible et tangible sa présence aux yeux des chanoines qui processionnaient devant elle à deux reprises au cours de l'année ${ }^{18}$. Le nombre de ces chanoines était probablement important à ces occasions, comme le suggèrent les rares fragments de comptes de la fabrique de la cathédrale conservés, notamment celui de l'année 1530-1531, soit chronologiquement proche de la mort de l'évêque. Le document signale en moyenne 20 à 22 chanoines - sur les trente chanoines que compte la cathédrale - se présentant en procession sur les

15. Cf. C. Huguenin, G. Cassina, D. Lüthi (dir.), Destins de pierre, p. 77-86; K. Berclaz, Les voies de l'éternité, p. 169-185.

I6. C. Huguenin, G. Cassina, D. Lüthi (dir.), Destins de pierre, p. 81 et 162.

17. Ibid.

I8. Cf. J. Gremaud, "Nécrologe de l'église cathédrale de Lausanne», p. 99 (14 janvier) et 227 (jour de la Sainte-Trinité). Les douze célébrations de l'évêque ne sont pas consignées dans le nécrologe, de sorte que l'on ne sait pas si d'autres processions avaient lieu à d'autres dates. 
tombes des grands dignitaires ecclésiastiques ${ }^{19}$. Il n'y est pas fait mention du tombeau d'Aymon de Montfalcon, mais l'importance d'un tel prélat autorise à déduire qu'une assistance de choix rehaussait le lustre donné à ces cérémonies. Nos recherches ont d'ailleurs montré que lors de ces occasions, la présence des chanoines est très recherchée autour des monuments épiscopaux ${ }^{20}$.

Un tel phénomène de visibilité se prolonge et s'exprime plus largement dans le décor et les legs réalisés au profit de la chapelle. Notre attention se porte ici spécialement sur ce qui procède de façon certaine de l'initiative de l'évêque. Il peut ainsi s'agir non seulement du matériel liturgique nécessaire aux célébrations commémoratives, mais aussi de tout ornement participant au lustre de la chapelle. Ces différents éléments contribuent à la logique de l'entretien du souvenir d'un personnage important. Une grande partie du mobilier a aujourd'hui disparu, mais il est bien décrit dans la visite de 1529, qui insiste par ailleurs sur la qualité des différents objets, dont les nombreux qualificatifs ("somptueux", "honorablement», "beau ", "décent») dépassent de loin ceux attribués aux autres autels.

Parmi les exemples les plus marquants, il convient de citer les stalles conservées et disposées dans la chapelle dès 1509. Celles-ci multiplient les références à l'évêque, par le biais de ses armoiries, de sa devise, de ses initiales ou encore de petits faucons sur le sommet des stalles ${ }^{21}$. Par deux fois, Aymon est même représenté en fondateur à genoux devant saint Maurice et ses compagnons, patrons de la chapelle, ainsi que devant la Vierge et présenté par ses saints patrons Jean-Baptiste et Benoît.

Gravée, sculptée, brodée ou encore peinte, la présence d'Aymon de Montfalcon est largement diffusée sur l'ensemble de sa chapelle et sur tous types de supports: la façade donnant sur le vestibule a conservé la devise et les armoiries de l'évêque au-dessus de la porte en noyer accompagnée de la date de 1504 qui marque l'année de la fondation de la chapelle ${ }^{22}$; deux fenêtres décorées de vitraux figuraient à l'origine les

I9. ACV, Aa 7/28, n 3353 (cf. p. ex. fol. 100v-101r et 104r-v).

20. K. Berclaz, Les voies de l'éternité, p. 135-138 et 201-225. Le nécrologe de la cathédrale confirme cette observation, cf. J. Gremaud, "Nécrologe de l'église cathédrale de Lausanne», passim.

2I. Sur la représentation constante d'Aymon de Montfalcon, cf. plus largement l'article de B. Pradervand dans ce volume.

22. ACV, Ac 15/2, fol. 230r (cf. supra, n. 9). 
armes de l'évêque et celles de ses parents ${ }^{23}$; enfin, un calice et une chasuble de camelot violet étaient timbrés de ses armoiries ${ }^{24}$. L'attention portée à l'identité montre l'effort particulier du défunt pour investir pleinement les lieux.

\subsection{Les célébrations commémoratives}

À l'image de ses prédécesseurs, Aymon de Montfalcon suit une tendance largement observée à Lausanne, consistant à fonder des messes commémoratives, répétées chaque année sur le principe de la perpétuité et moyennant un capital qui servira à financer ces célébrations par le versement d'un cens annuel ${ }^{25}$. Le cens, ou la rente, correspond donc au prix annuel d'une messe. La notion de perpétuité est ici toute relative, car le système des messes disparaît lors du passage à la Réforme en 1536. Cependant, ces célébrations - aussi appelées messes-anniversaire - sont inscrites dans le nécrologe de la cathédrale; elles figurent donc toujours dans le livre commémoratif grâce auquel nous connaissons leur nombre. Ainsi, le 10 août, jour de sa mort, des vigiles et une messe sont célébrées et des distributions sont faites aux chanoines "pour l'un des douze anniversaires fondés par lui " ${ }^{26}$. Un deuxième anniversaire figure au 10 novembre, pour lequel on procède comme le 10 août ${ }^{27}$. L'intérêt de ces notices réside dans la répétition, et surtout dans la régularité des messes: chaque $10 \mathrm{du}$ mois a lieu une nouvelle célébration. Le premier anniversaire a lieu au jour de sa mort, suivi de deux anniversaires au 10 septembre et au 10 octobre, mais qui ne figurent pas dans le nécrologe. Cette logique est confirmée le 10 novembre, puisque le

23. Ibid., fol. 230v: Tamen aliunde sunt ibi in dicta capella fenestre duplices cum verreriis sumptuosis, pulcris ymaginibus, cum armis dicti domini fondatoris et suorum parentum.

24. Ibid., fol. 230v-231r: Reperti fuerunt duo calices argentei cum patenis suis etiam argenteis [...], altero calice magis alto cum armis fondatoris in pede desuper [...]. Item una casula de camellotto violeto, foderata tela nigra, cum cruce de brodura ex filo aureo retro, et colona ante tantum, in qua sunt impressa arma domini fondatoris ab utraque parte [...].

25. Sur cette pratique systématique chez les évêques de Lausanne, cf. K. Berclaz, Les voies de l'éternité, en part. p. 201-225.

26. J. Gremaud, «Nécrologe de l'église cathédrale de Lausanne», p. 171.

27. Ibid., p. 207 (ut supra $X^{\text {ma }}$ augusti). 
document indique ici qu'il s'agit du quatrième anniversaire célébré pour l'évêque.

Dans les deux notices du nécrologe, la distribution aux chanoines est fixée à 60 sous, soit un cens ou une rente annuelle de 3 livres par messe. Nos recherches ont montré qu'à la fin du Moyen Âge, en Europe et à Lausanne particulièrement, le prix annuel d'une messe revient le plus souvent au $5 \%$ du capital investi ${ }^{28}$. Ici, le capital s'élèverait donc à 60 livres par messe. Les sources capitulaires conservées le confirment: le 19 août 1517, peu après la mort d'Aymon, le manual stipule que l'évêque a réservé aux chanoines un capital de 1200 florins de Savoie pour les douze messes-anniversaire ${ }^{29}$, soit 100 florins par messe qui équivalent précisément à 60 livres $^{30}$. Bien que peu élevé, le cens annuel prévu ici (3 livres) correspond au prix d'une messe lausannoise, qui varie entre 3 et 8 livres pour un évêque, l'important n'étant pas nécessairement un montant élevé, mais bien plus une répétition assurée sur l'ensemble de l'année ${ }^{31}$. Avec ses douze messes soigneusement réparties, Aymon répond parfaitement à cette stratégie commémorative.

Le manual précise au 19 août 1517 l'ajout vraisemblable de quatre messes-anniversaire supplémentaires pour un total de 400 florins de Savoie, soit à nouveau un capital de 60 livres par messe ${ }^{32}$. Au total, l'évêque investirait donc dans seize commémorations annuelles, un nombre très important et largement supérieur à ses prédécesseurs. Ces célébrations lui assurent une grande notoriété puisqu'il s'agit de messes publiques dont l'apparat est conséquent, qui reproduisent à une échelle plus modeste la cérémonie de funérailles. Or, nous l'avons souligné, celles-ci sont réalisées de manière honorable ${ }^{33}$. Il faut donc imaginer qu'elles bénéficiaient parfois de processions, avec une assistance de

28. K. Berclaz, Les voies de l'éternité, p. 207.

29. ACV, Ac 13, fol. 237v: Et quod per presens prefatos dominos requisiverat quatenus modo premisso eamdem fundationem auctorizatione dignaretur, quodque pro duodecim anniversariis dabat prefatis dominis duodecim centum florenos Sabaudie pro semel.

30. À la fin du Moyen Âge à Lausanne, le florin vaut 12 sous de monnaie locale. Pour obtenir une livre de monnaie de compte, 20 sous sont nécessaires, donc un florin équivaut à 0,6 livre. Cf. K. Berclaz, Les voies de l'éternité, p. 40, n. 113.

3I. Ibid., p. 248.

32. ACV, Ac 13, fol. 237v: Et dominis celebrantibus dicte ecclesie, pro supportatione alicuius oneris et quatuor anniversariis, videlicet quatuor centum florenos similes pro semel.

33. Cf. supra, p. 244. 
choix et l'utilisation d'un luminaire important, ainsi que des récitations particulières. Les sources sont le plus souvent avares en descriptions liturgiques, de sorte quaucune indication concernant Aymon de Montfalcon n'a été retrouvée, mais l'observation d'autres cas épiscopaux permet de suggérer qu'il en va de même pour un prélat de cette envergure ${ }^{34}$. L'ensemble des messes met donc en lumière la qualité exceptionnelle du défunt avec une série de gestes pensés spécifiquement pour lui, et dans un contexte architectural totalement conçu pour rappeler son identité.

Parallèlement aux commémorations annuelles, certains évêques fondent encore des chapellenies, à savoir des messes régulières et hebdomadaires, voire quotidiennes; elles sont aussi basées sur le principe de la perpétuité et répétées sur l'ensemble de l'année, mais ces célébrations ne sont pas indiquées dans le nécrologe. Il s'agit cette fois de messes privées, récitées à voix basse par un ou plusieurs chapelains. Le caractère public disparait donc au profit de l'intimité de la chapelle. Cette célébration est assurée ici par quatre chapelains chargés de réciter deux messes par jour avec récitations liturgiques à la fin de chaque messe, comme l'indique l'acte de fondation de la chapellenie:

[...] fundamus, erigimus et dotamus unam capellaniam [...] in prefata ecclesia, videlicet in campanili sito prope anteriores fores eiusdem ecclesie a parte superiori et borea, in honore et sub invocatione sanctorum martirum Mauricii, Secundi, Candidi, Victorum (sic) et sociorum suorum totiusque sacre legionis Thebee predicte denominandam necnon et quattuor capellanos sive rectores [...], qui, et eorum successores, omni die duas missas submissa voce [...] celebrare tamen dum tempus affuerit alta voce celebrandas et divine qualibet expleta missa psalmum "De profundis» cum colectis "Deus qui inter apostolicos, sacerdotes et fidelium etc." super tumuro (sic) sive sepultura nostra in eadem capella fienda dicere obligantur, et ad hoc sunt adstricti ${ }^{35}$.

Notons ici le nombre particulier de messes basses, qui témoignent à nouveau du prestige du défunt. Nos recherches sur les évêques de Lausanne indiquent une fréquence maximale d'une messe quotidienne, quel que

34. Sur les gestes funéraires des évêques, cf. K. Berclaz, Les voies de l'éternité, p. 124146.

35. ACV, C Vb 993, p. 2-3. 
soit le défunt ${ }^{36}$. Par ailleurs, les récitations qui ponctuent ces messes renforcent la valeur salvatrice attribuée aux célébrations réalisées dans le cadre privé et "en présence» de l'évêque, puisque la chapellenie doit se faire sur son tombeau. Dans la liturgie, le De profundis est un psaume pénitentiel faisant partie des prières pour les morts, tandis que le Deus qui inter apostolicos est une oraison prononcée lors de l'office des morts ${ }^{37}$.

La visite de 1529 confirme les modalités souhaitées par l'évêque. Elle montre qu'après sa mort, ses volontés sont suivies à la lettre, ou pratiquement. En effet, si le nombre de chapelains reste identique, le nombre de messes quotidiennes, lui, s'élève désormais à quatre. Il est possible qu'il s'agisse d'une erreur du scribe. Le chapelain interrogé par les commissaires lors de la visite indique en tous les cas ne pas avoir vu l'acte de fondation ${ }^{38}$. Cependant, les indications quant aux redevances coïncident parfaitement avec l'acte de 1517, de sorte que nous pensons à une réelle augmentation du nombre de messes, ajoutant un surcroît d'importance au prélat douze ans après sa mort. Assurément, Aymon de Montfalcon est l'évêque qui se distingue le plus parmi les dignitaires de l'Église de Lausanne en remplissant l'année liturgique de son souvenir.

L'étude des différents moyens matériels et immatériels mobilisés dans la chapelle d'Aymon de Montfalcon montre ainsi le cumul des possibilités de "faire mémoire» par les multiples cérémonies, mais également de manière très tangible par l'impact visuel assuré par la tombe, par le décor et par les legs de l'évêque.

\section{L'espace ecclésial et la chapelle d'Aymon de Montfalcon}

À Lausanne, les sépultures épiscopales privilégient des lieux très fréquentés en raison d'un pèlerinage dédié à la Vierge qui s'organise dès le début du XIII ${ }^{e}$ siècle. Leur répartition entre le XII ${ }^{e}$ et le début du XVI ${ }^{e}$ siècle indique une nette concentration dans la partie orientale de la cathédrale. Elles semblent suivre un parcours, que Werner Stöckli a proposé comme

36. Nous préparons actuellement une thèse de doctorat sur la cathédrale de Lausanne à la fin du Moyen Âge et sur les multiples manifestations de piété liées à celle-ci.

37. J. Avril, "La paroisse médiévale et la prière pour les morts», p. 57 sq.

38. ACV, Ac 15/2, fol. 232r. 
hypothèse pour les chanoines (voir pl. X) ${ }^{39}$. Il se peut que les pèlerins l'aient emprunté aussi, du moins en partie. On sait qu'ils avaient accès au bras nord du transept, et que de là, ils traversaient à genoux le déambulatoire vers la chapelle de la Vierge dans le bras sud du transept, point d'orgue et destination du pèlerinage. Hans von Waltheim, bourgeois et marchand de Halle, décrit en 1474 un tel parcours par une femme dans son carnet de voyage ${ }^{40}$. Il faut donc supposer un chemin passant par le bras nord du transept et le déambulatoire, avec une éventuelle utilisation préalable des galeries du cloître, avant de parvenir à la chapelle de la Vierge. Cette chapelle accueille dès le XIII e siècle des reliques de la Vierge et une statue mariale réputée miraculeuse. Les tombes sont ainsi positionnées en fonction des différentes circulations et utilisations de l'espace ecclésial ${ }^{41}$.

Si cette répartition ne souffre aucune discussion jusque vers la fin du $\mathrm{XV}^{\mathrm{e}}$ siècle, la logique est perturbée dès le début du siècle suivant avec la fondation de la chapelle d'Aymon de Montfalcon dans la tour nord inachevée du massif occidental en $1504^{42}$. Quelles raisons poussent alors subitement un évêque à aménager une chapelle fastueuse, mais reléguée bien au-delà du pôle oriental et de la vie liturgique centrée sur la chapelle de la Vierge? L'éloignement de cette "constellation" de sépultures trouve une explication probable dans les importantes transformations d'Aymon au tout début du XVI ${ }^{e}$ siècle dans le massif occidental où vient s'insérer la chapelle. Marcel Grandjean a étudié avec précision ces réaménagements ${ }^{43}$. Nous savons donc que la fermeture de la "Grande travée» entre 1504 et 1505 et la destruction d'un portail du XIII ${ }^{\mathrm{e}}$ siècle dans le magnum portale aura pour conséquence d'harmoniser et d'homogénéiser tout l'espace de la cathédrale. Jusqu'alors, la zone du magnum portale n'était pas consacrée dans sa partie inférieure. Parallèlement, l'évêque

39. W. Stöckli, "Les fouilles archéologiques entreprises au nord de la cathédrale», p. 47.

40. F. E. Welti, Die Pilgerfahrt des Hans von Waltheym im Jahre 1474, p. 14 sq.

4I. Sur l'agencement de ces tombes et leur lien avec la chapelle de la Vierge, cf. K. Berclaz, Les voies de l'éternité, p. 151-169 et «Le transept de la cathédrale de Lausanne (XII $-X V I^{\mathrm{e}}$ siècle)", p. 130 sq.

42. Cf. supra, n. 3.

43. M. Grandjean, «Le magnum portale de la cathédrale de Lausanne et le passage routier de la "grande travée" " et "La cathédrale actuelle», p. 51-56 et 125-139. Cf. aussi les contributions de K. Queijo, de M. Grandjean et de D. Lüthi dans ce volume. 
libère de l'espace au nord du massif occidental, permettant ainsi le passage des chars et de la foule au-devant du massif où sera entamée dès 1515 la construction du portail monumental de l'évêque (voir pl. X) ${ }^{44}$.

Dès le début du XVI ${ }^{\mathrm{e}}$ siècle, l'évêque, de concert avec le chapitre, a cherché à faire de cette zone occidentale la nouvelle entrée principale de la cathédrale. Désormais, l'accès à la chapelle de la Vierge est plus axial, soit d'ouest en est, alors que jusqu'à cette date, l'entrée principale était encore assurée par le portail peint érigé sur le flanc sud de la cathédrale, présentant alors l'avantage d'être dirigé vers la porte Saint-Étienne d'où pouvaient arriver les pèlerins. Il semble par ailleurs que la disposition des trois statues au niveau de l'arcade du narthex (fig. 1) ait pu être une initiative d'Aymon de Montfalcon. En réutilisant des sculptures en provenance supposée du portail du XIII e siècle, l'évêque aurait cherché à marquer l'entrée de la cathédrale par un ensemble qui convient bien au seuil d'un édifice religieux et à la fonction d'accueil qu'on lui prête ${ }^{45}$. En effet, il profite de la présence de dais pour y placer les rondes-bosses représentant le roi Salomon, la reine de Saba et la Vierge à l'Enfant sur un trône de Sagesse ${ }^{46}$. Ce schéma trinitaire assez classique renvoie à l'image de la victoire de l'Église: la reine de Saba est une préfiguration vétérotestamentaire de l'Église; la Vierge est souvent perçue comme son incarnation, avec un discours qui s'intensifie au XII siècle ${ }^{47}$, tandis que le trône de Sagesse sur lequel elle siège est volontiers associé à la sagesse exemplaire du roi Salomon. Cette figuration victorieuse remplit alors pleinement sa fonction d'accueil une fois le seuil du nouveau portail monumental franchi, et remplace avantageusement celle qu'occupait jusqu'alors le portail peint et son iconographie dédiée à la Vierge et au Christ. Ici, le programme est centré sur la Vierge en figure ecclésiale, accueillant la communauté des fidèles en son sein et préfigurant la

44. Sur la chronologie précise de la construction de ce portail et sur les nombreuses délibérations que celui-ci a suscitées, nous renvoyons à l'article de K. Queijo dans ce volume.

45. Cet exemple de réemploi complète bien, selon nous, les exemples fournis par D. Lüthi à propos des récupérations que connaît la cathédrale au fil de ses réaménagements depuis le milieu du XII ${ }^{\mathrm{e}}$ siècle et auxquelles s'adonne particulièrement Aymon de Montfalcon.

46. P. Kurmann (éd.), La cathédrale Notre-Dame de Lausanne, en part. p. 129-133.

47. Sur ce discours, cf. en premier lieu M.-L. Thérel, Le triomphe de la Vierge-Église et D. Iogna-Prat, É. Palazzo, D. Russo (dir.), Marie. 
Fig. 1 - Grande entrée de la cathédrale de Lausanne, deuxième arcade et claustra donnant sur la tribune avec la statue fragmentaire de la Vierge à l'enfant. Photographie Jeremy Bierer, 2012. 
présence de la statue mariale à l'autre bout de l'église, dans la chapelle de la Vierge.

Avant même la fin des travaux du massif occidental se manifeste le souci de créer un espace consacré sur l'ensemble de l'édifice par une petite agglomération de chapelles privatives entre 1500 et 1504 , entre l'emplacement actuel du portail Montfalcon et la "Grande travée». Il s'agit des chapelles des chanoines Guillaume Colombet et Pierre Flory, fondées respectivement avant le 7 octobre $1500^{48}$ et le 10 novembre $1502^{49}$ (voir pl. X). L'intégration de ces fondations privées à l'espace consacré a cependant mis du temps à se concrétiser puisque l'emplacement définitif du portail de l'évêque censé fermer l'édifice n'était pas encore établi en $1515^{50}$, alors que les délibérations entre le chapitre et l'évêque remontent en tout cas à 1504 . Les préoccupations des chanoines sont d'ailleurs clairement perceptibles lors de la séance capitulaire du 25 mars 1504, où les dignitaires insistent auprès de l'évêque pour que ce portail soit érigé à l'extrémité occidentale de l'église «afin d'y intégrer les chapelles nouvellement fondées par les chanoines Guillaume Colombet et Pierre Flory " ${ }^{51}$.

Si l'évêque a longtemps souhaité construire ce portail plus à l'intérieur du massif pour des raisons probablement financières, les faits nous montrent que le chapitre reste maitre en sa cathédrale et que la décision finale lui revient. C'est dire surtout la valeur que l'on attribuait alors à cette zone préparée pour devenir un nouveau pôle sacré au moment où Aymon de Montfalcon était sur le point de fermer le passage routier de la "Grande travée»: sans attendre l'achèvement des travaux, de nouvelles chapelles y sont fondées, auxquelles viendra rapidement s'ajouter celle de l'évêque. Nous comprenons dès lors pourquoi cette fondation, à première vue très éloignée de l'activité liturgique de l'église, est en réalité aménagée dans l'un des lieux les plus importants et les plus en vue au

48. Chapelle dédiée aux saints Joseph et Félix mentionnée dans le testament de Guillaume Colombet en date du 7 octobre 1500, dans lequel elle est déjà qualifiée de noviter fondata. Cf. ACV, C Va 2363.

49. Chapelle dédiée à la Passion et aux saints Pierre et Paul, selon l'acte de fondation du 10 novembre 1502. Cf. ACV, C Vb 859.

50. ACV, Ac 13, fol. 173r (vendredi 2 mars 1515). Sur les nombreuses négociations entre le chapitre et l'évêque quant à l'emplacement du portail et le retard de sa construction, voir la contribution de K. Queijo dans ce volume.

5I. ACV, Ac 13, fol. 3r-v. 
début du XVI ${ }^{\mathrm{e}}$ siècle, à l'entrée immédiate de celle-ci et bien visible des pèlerins grâce à ses systèmes d'ouverture (guichet sur la façade du côté du vestibule et grilles du côté de la nef permettant de voir l'intérieur de la chapelle) et à ses marquages visuels par le biais des armoiries et devise personnelle largement ventilées sur l'ensemble des interventions de l'évêque dans le massif occidental.

Lactivité des pèlerins entérine définitivement la position stratégique occupée par la chapelle au sein de l'édifice. Elle est à lier au développement des indulgences, aussi appelées Grand Pardon, obtenues par les chanoines en 1450 et ayant lieu tous les sept ans, trois jours consécutifs durant la Semaine sainte de Pâques ${ }^{52}$. L'importance de ce jubilé est bien illustrée par l'une des notices du manual du chapitre en date du vendredi 17 mars 1514, qui signale que, depuis que ces indulgences existent, le beffroi qui porte les cloches dans la tour sud du massif - donc à proximité de la chapelle et du portail Montfalcon - a connu un net regain d'intérêt et a suscité des largesses de la part des pèlerins à tel point que le maître de la fabrique entend le détruire complètement pour le reconstruire à neuf ${ }^{53}$.

Le Grand Pardon était annoncé à Lausanne sur des pierres similaires à des tableaux qui contenaient les textes des indulgences. Or, en 1514, année de jubilé, deux tableaux sont signalés dans la cathédrale, l'un dans le chœur liturgique et l'autre dans la chapelle Montfalcon, devenue le nouveau pôle sacré de la cathédrale et visible des pèlerins venus en masse! Cette attention accrue portée à l'entrée occidentale est très perceptible dans le manual du chapitre dans les semaines qui précèdent la semaine pascale et doit être mise en relation directe avec le Grand Pardon. On y discute à plusieurs reprises de la construction du portail ${ }^{54}$.

52. Sur le système des indulgences ou du Grand Pardon, cf. E. Dupraz, La cathédrale de Lausanne, p. 354-360 et surtout J.-D. Morerod, «Entre soumission à Rome et velléités identitaires".

53. ACV, Ac 13, fol. 323v: Tandem dominus Johannes de Bargo, magister fabrice, de voluntate dictorum dominorum capitulantium, dixit se velle facere descendere magnum simballum integrum deorsum de simballatorio, quia quando lucrabuntur indulgentie, istud simballum visitabitur ab omnibus visitantibus ecclesiam, et erit maior devotio facienda et largienda de bonis suis pro reparatione illius simballi.

54. Le 31 mars 1514, le chanoine Louis du Pas est envoyé au château Saint-Maire auprès de l'évêque malade afin de le supplier d'avancer la construction du grand portail (ACV, Ac 13, fol. 326v). Peu de temps auparavant, le 21 février 1514, une missive du pape Léon X est envoyée aux évêques de Sion et de Belley condamnant sévèrement la 
C'est dans ce contexte particulier que s'insère le projet de reconstruction du clocher à l'entrée de la cathédrale, un mois avant le début des indulgences. De toute évidence, le chapitre souhaite activement réorganiser l'espace ecclésial en fonction du pèlerinage et réserver la part belle à la chapelle de l'évêque. À l'aube de nouveaux usages liturgiques, les adaptations nécessaires profiteront ainsi pleinement à cette fastueuse fondation épiscopale.

On sait désormais la manière dont Aymon de Montfalcon concevait sa mémoire à l'intérieur des murs de sa chapelle privative et comment celleci s'est insérée au sein de l'espace ecclésial. Les résultats montrent que cet évêque compte parmi les grands dignitaires lausannois. Son comportement suit les tendances de ses prédécesseurs par une fondation funéraire et privative fastueuse réunissant de nombreux médias et moyens pratiques. La chapelle de saint Maurice et des martyrs thébains s'ancre alors dans la cathédrale et représente l'une des plus fastueuses fondations, si ce n'est la plus importante de l'histoire de cette cathédrale. Elle dépasse en somptuosité nombre d'entre elles et a peut-être été prévue un temps pour accueillir une liturgie spécifique ${ }^{55}$. Dès sa fondation, elle fait partie intégrante de l'espace ecclésial et de sa liturgie qui évolue sensiblement au début du XVI e siècle et qui semble étroitement liée au développement des indulgences dès le milieu du XVe siècle.

Le cas d'Aymon de Montfalcon reste probablement le plus emblématique et le plus homogène au niveau de la documentation, mais il montre quaujourd'hui encore la mémoire épiscopale lausannoise représente un formidable terrain d'enquête.

Kérim Berclaz

Université de Lausanne

négligence d'Aymon de Montfalcon qui a laissé l'entrée à l'abandon depuis quinze ans (traduction partielle dans E. Dupraz, La cathédrale de Lausanne, p. 489 sq., qui date toutefois le bref du pape de l'année 1513 par erreur).

55. C'est l'hypothèse d'une chapelle collégiale formulée par D. Lüthi dans ce volume. 


\section{BIBLIOGRAPHIE}

Avril, Joseph, "La paroisse médiévale et la prière pour les morts", in L'Église et la mémoire des morts dans la France médiévale. Communications présentées à la table ronde du C.N.R.S., le 14 juin 1982, réunies par Jean-Loup Lemaître, Paris, Études augustiniennes, 1986, p. 53-68.

Berclaz, Kérim, Les voies de l'éternité. Les testaments des évêques de Lausanne et la construction d'une mémoire épiscopale (XIV $-X V^{e}$ s.), Lausanne, Université de Lausanne, 2017 (Cahiers lausannois d'histoire médiévale, 57).

—, "Le transept de la cathédrale de Lausanne (XII ${ }^{\mathrm{e}} \mathrm{XVI}{ }^{\mathrm{e}}$ siècle) : un pivot essentiel à la vie religieuse", in Le transept et ses espaces élevés dans l'église du Moyen Âge (XIe-XVIe siècles). Pour une approche fonctionnelle (architecture, décor, liturgie, son). Actes du colloque de Lausanne (20-21 avril 2015), éd. par Barbara Franzé, Nathalie Le Luel, Zagreb, University of Zagreb/International Research Center for Late Antiquity and the Middle Ages, 2018, p. 125-138.

Coutaz, Gilbert, "L'inventaire de 1394 des archives de l'évêque de Lausanne: contribution à une histoire archivistique du Pays de Vaud", Revue historique vaudoise, 109 (2001), p. 89-132.

Dupraz, Emmanuel, La cathédrale de Lausanne. Etude historique, Lausanne, Librairie Th. Sack, 1906.

Grandjean, Marcel, «La cathédrale actuelle. Sa construction, ses architectes, son architecture", in La cathédrale de Lausanne, éd. par Jean-Charles Biaudet et al., Berne, Société d'histoire de l'art en Suisse, 1975, p. 45-174.

—, "Le magnum portale de la cathédrale de Lausanne et le passage routier de la "grande travée" ", Revue suisse d'art et d'archéologie, 32/3 (1975), p. 193-220.

Gremaud, Jean, "Nécrologe de l'église cathédrale de Lausanne", Mémoires et documents publiés par la Société d'histoire de la Suisse romande, 18 (1863), p. 89-246. 
Huguenin, Claire, Cassina, Gaëtan, Lüthi, Dave (dir.), Destins de pierre. Le patrimoine funéraire de la cathédrale de Lausanne, Lausanne, Cahiers d'archéologie romande, 2006 (Cahiers d'archéologie romande, 104).

Iogna-Prat, Dominique, Palazzo, Éric, Russo, Daniel (dir.), Marie. Le culte de la Vierge dans la société médiévale, Paris, Beauchesne, 1996.

Kurmann, Peter (éd.), La cathédrale Notre-Dame de Lausanne. Monument européen, temple vaudois, Lausanne, La Bibliothèque des arts, 2012.

Morerod, Jean-Daniel, Genèse d'une principauté épiscopale. La politique des évêques de Lausanne (IXe-XIVe siècle), Lausanne, Bibliothèque historique vaudoise, 2000 (Bibliothèque historique vaudoise, 116).

—, "Entre soumission à Rome et velléités identitaires. Le Grand Pardon de Lausanne (1450-1534)», Revue historique vaudoise, 119 (2011), p. 35-58.

Plantin, Jean-Baptiste, De l'antiquité de la ville de Lausanne, Manuscrit dit de Dompierre, (s. l.) [Lausanne], 1656. BCU, Ms., F. 1071.

Reymond, Maxime, "Aymon de Montfalcon, évêque de Lausanne, 1491-1517", Revue d'histoire ecclésiastique suisse, 14 (1920), p. 28-39 et 99-111.

STÖсKLI, Werner, «Les fouilles archéologiques entreprises au nord de la cathédrale», in Le cloître de la cathédrale Notre-Dame de Lausanne, éd. par Marcel Grandjean, Werner Stöckli, Pierre Margot, Claude Jaccottet, Lausanne, Bibliothèque historique vaudoise, 1975, p. 23-66 (Cahiers d'archéologie romande, 4).

Thérel, Marie-Louise, Le triomphe de la Vierge-Église. À l'origine du décor du portail occidental de Senlis. Sources historiques, littéraires et iconographiques, Paris, Éd. du CNRS, 1984.

Welti, Friedrich Emil, Die Pilgerfahrt des Hans von Waltheym im Jahre 1474, Berne, Stämplli, 1925. 